\title{
Adaptación de Alumnos según estilos de Afrontamiento Individual al Primer año de la Carrera de Arquitectura
}

(Estudio de caso)

Autor: Omar Eduardo Cañete Islas Universidad de Valparaíso, UV ocanetei00@yahoo.es Valparaíso, Chile

\section{Resumen}

El desafío y aporte en esta propuesta es aplicar, mediante técnicas de auto reporte, tanto una clasificación del estilo primario y secundario de afrontamiento, en un contexto de desempeño grupal de alumnos de primer año de la carrera de arquitectura de la Universidad de Valparaíso, en el marco de los criterios y objetivos de autorregulación promovidos por la Universidad a través de la Unidad de división Académica. Con esto, se espera obtener medidas cuantitativas y cualitativas de la evolución individual y cambios en los procesos de autorregulación del aprendizaje, asociado a la experiencia de trabajo grupal.

Palabras clave: arquitectura; aprendizaje; creatividad; trabajo grupal. 


\title{
Adaptation of Students According to Individual styles in First year of Architecture \\ (Case study)
}

\begin{abstract}
The challenge and contribution in this proposal is to apply, through selfreport techniques, both a classification of the primary and secondary style of coping, in a context of group performance of first year students of the architecture degree at the University of Valparaiso, in the framework of selfregulation criteria and objectives promoted by the University through the Academic Division Unit.
\end{abstract}

Keywords: architecture; learning; creativity; group work. 
"ELENA: ... Y por ella se dice que el amor es niño, siendo tan a menudo engañado en la elección. Y como en sus juegos perjuran los muchachos traviesos, así el rapaz amor es perjurado en todas partes; pues antes de ver Demetrio los ojos de Hermia me juró de rodillas, que era sólo mío; más apenas sintió el calor de su presencia, deshiciéronse sus juramentos como el granizo al sol...". (Sueño de una noche de verano; William Shakespeare).

\section{Antecedentes}

Desde hace unos años, la Universidad de Valparaíso, ha dispuesto la implementación de cursos transversales de autorregulación del estudio para alumnos de primer año de todas sus carreras. Esto tanto con el fin de entregar herramientas de adaptación a la vida universitaria, como para evitar niveles crecientes de deserción por problemas de adaptación académica, como con el interés de crear un cierto sello o perfil formativo en sus alumnos. En el caso de la Escuela y carrera de Arquitectura, este proceso, ha tenido algunas particularidades y dificultades, por razones tales como:

1. La necesidad de incentivar un perfil creativo por parte de los alumnos.

2. La necesidad, por ende, que cada alumno desarrolle su propio proceso de descubrimiento propio de potencialidades, y no sólo un aprendizaje pasivo de contenidos.

3. La dificultad encontrada e los alumnos de realizar encargos grupales, donde cada uno pueda aportar creativamente a la solución de encargos colectivos.

4. Hasta cierto punto, se ha encontrado que los puntos anteriores entran en conflicto, pues el desarrollo creativo aparece como un elemento percibido principalmente como rasgo individual.

5. Lo anterior se ve reforzado, pues los alumnos que ingresan son sobre 100 a 120 alumnos por año, lo que obstaculiza evaluaciones y seguimientos individuales. Esto redunda en que se produzcan niveles de 
competencia no esperados, en la discusión de ideas creativas, y cómo éstas son puestas en juego por el equipo, para llevarlas a un buen término.

\section{Marco Teórico}

En el marco de las relaciones interpersonales se define el conflicto como un proceso dinámico que ocurre entre personas que sufren reacciones emocionales negativas al percibir discrepancias e interferencias en el logro de sus objetivos

La percepción negativa de la vivencia, la falta de habilidades para identificar las emociones y la incapacidad para afrontar la comunicación asertivamente, en definitiva, los estilos de afrontamiento que la persona dispone para gestionar las discrepancias son determinantes en la evolución y desenlace del Conflicto, autores como Piorno (2012), discurren que los estilos influyen de modo directo en la aparición o disminución de fenómenos tales como es el conflicto, la pérdida de confianza del equipo, el debilitamiento de la red social y el fracaso en la consecución de objetivos de trabajo. Esta realidad del entorno se presenta como un elemento estresante que repercute negativamente en la satisfacción laboral del profesional pudiendo ello afectar a los subsistemas y sistema global de la organización.

Concordantemente, la satisfacción laboral ha sido definida como un estado emocional positivo o placentero resultante de la percepción subjetiva de las experiencias que predispone a la persona a una actitud ante el trabajo. En esta línea, se ha demostrado que la insatisfacción del personal se correlaciona negativamente con la productividad expresada por el absentismo, la rotación, el incremento de quejas de pacientes y el deterioro en el logro de objetivos, repercutiendo en los costes indirectos que debe soportar la organización. Desde esta perspectiva y a partir de la Teoría de los Dobles Intereses diseñada por Kilmann (1974-1977), se sostiene la línea de investigación de este trabajo. 
Este autor identifica cinco estilos para afrontar situaciones susceptibles de desatar un conflicto y que subyacen en base a dos características fundamentales: el asertividad y la cooperación. Este autor establece que las personas escogen diferentes estilos para afrontar los conflictos basándose en dos intereses primarios: el interés propio y el interés por los demás, siendo independientes entre sí y variando su intensidad en un rango de débil a fuerte. El interés propio se refiere al grado en que las partes en conflicto se centran en sus propias necesidades, intereses, valores y creencias; mientras que el interés por los demás se refiere a la medida en que las partes en conflicto se centran en las necesidades, intereses, valores y creencias de sus oponentes. Lo anterior se resume en la siguiente tabla:

Tabla 1. Estilos de Afrontamiento, según Modelo de Thomas Kilmann.

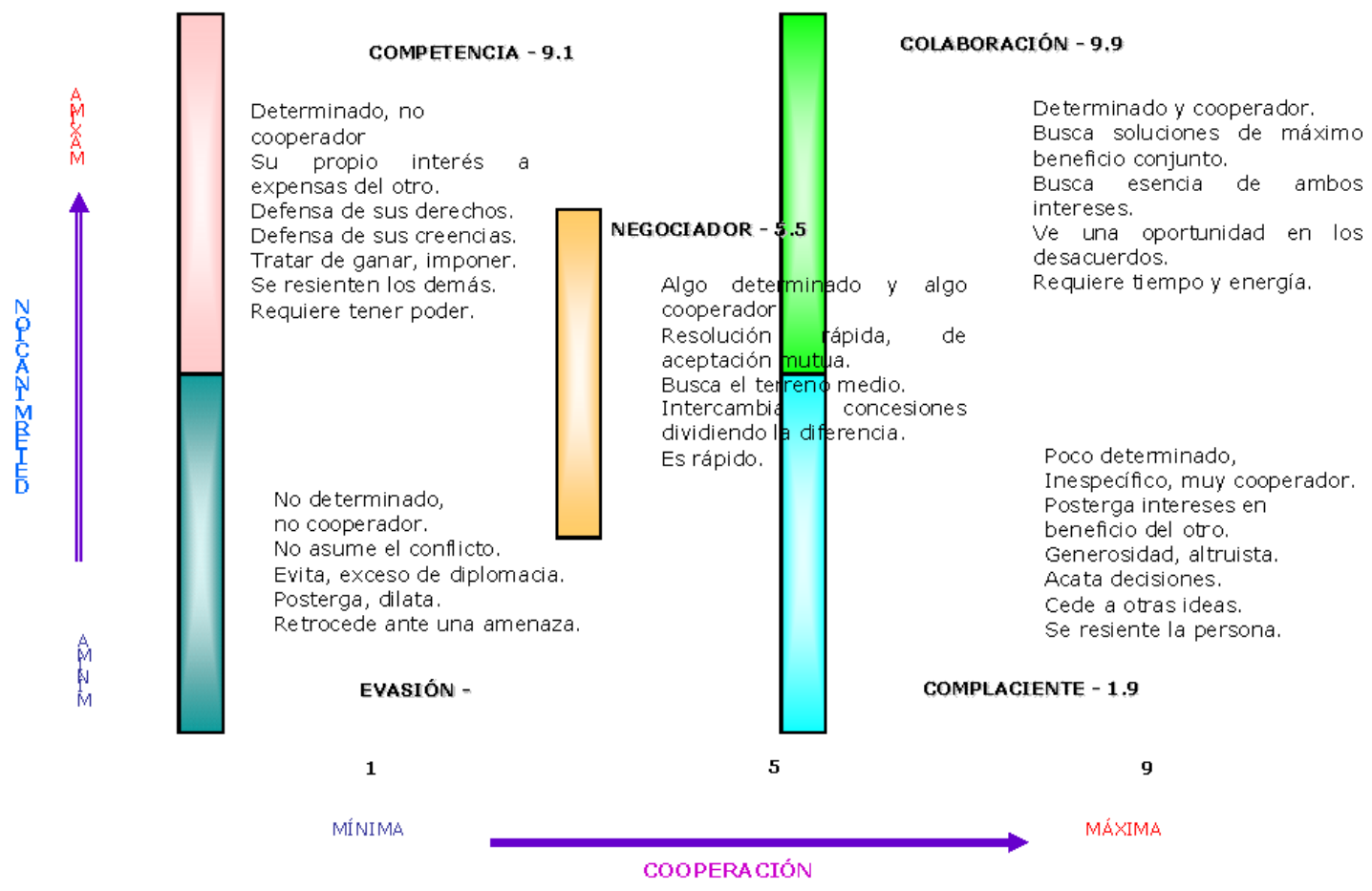

Fuente: Kilmann, (1974), Elaboración Propia, (2016).

El desafío y aporte en esta propuesta es aplicar, mediante técnicas de 
auto reporte, tanto una clasificación del estilo primario y secundario de afrontamiento, en un contexto de desempeño grupal de alumnos de primer año de la carrera de arquitectura de la Universidad de Valparaíso, en el marco de los criterios y objetivos de autorregulación promovidos por la Universidad a través de la Unidad de división Académica.

\section{Metodología}

A partir del marco anteriormente explicado, se les pidió a los alumnos, en base a técnicas de auto-reporte, guiado por preguntas específicas, realizaran una revisión y autovaloración del estilo de afrontamiento personal que cada uno desarrollo durante el semestre en el trabajo en equipo del taller, en donde tenían que coordinarse para hacer una propuesta general. En base a estas narraciones, se desarrolló un estudio de caso mixto, post-factum, tanto cuantitativo como cualitativo en base a un análisis de contenido de los relatos obtenidos, los que fueron tabulados según la descripción que cada uno realizaba de su experiencia. Se presentan entonces, los resultados, tanto desde el punto de vista de una estadística descriptiva como de contenidos en relación con los estilos de afrontamiento percibidos en su trabajo en grupos de taller.

\section{Resultados:}

4.1. Estadística Descriptiva y Análisis de Contenido: De los 83 casos evaluados, la composición muestral de los alumnos por sexo y su respectiva evolución de estilos de afrontamiento es la siguiente: 
Tabla Resumen. Evolución de los estilos primarios y secundarios.

\begin{tabular}{|l|l|l|l|l|l|}
\hline \multirow{2}{*}{$\begin{array}{l}\text { ESTILO } \\
\text { INICIAL }\end{array}$} & \multicolumn{2}{|c|}{ TOTAL } & \multirow{2}{*}{$\begin{array}{l}\text { ESTILO } \\
\text { SECUNDARIO }\end{array}$} & \multicolumn{2}{c|}{ TTOTAL } \\
\cline { 2 - 3 } & $\mathrm{N}$ & $\%$ & & $\mathrm{~N}$ & $\%$ \\
\hline Colaborador & 43 & 52 & Colaborador & 27 & 33 \\
\hline Evitativo & 17 & 21 & Evitativo & 20 & 24 \\
\hline Competitivo & 6 & 7 & Competitivo & 11 & 13 \\
\hline Negociador & 7 & 8 & Negociador & 13 & 16 \\
\hline Complaciente & 10 & 12 & Complaciente & 12 & 14 \\
\hline TOTAL & 83 & 100 & TOTAL & 83 & 100 \\
\hline
\end{tabular}

\begin{tabular}{|l|c|c|}
\hline Genero & N & $\%$ \\
\hline Femenino & 38 & 33 \\
\hline Masculino & 45 & 67 \\
\hline Total & 83 & 100 \\
\hline
\end{tabular}
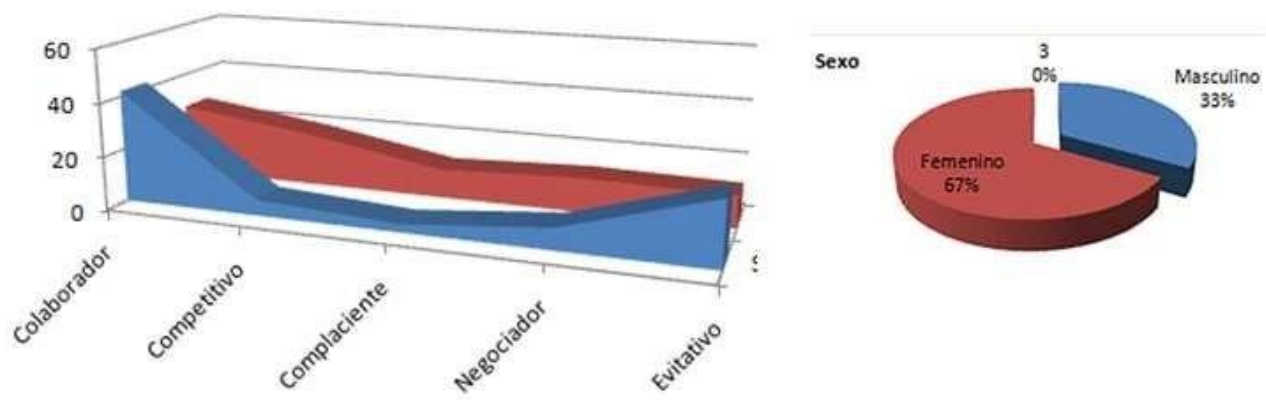

Fuente: Elaboración Propia (2016).

\subsection{Evolución de Estilos de Afrontamiento en el Semestre.}

\subsubsection{Estilo Colaborativo.}

4.2.1.1. Análisis Descriptivo: Desde el punto de vista de la evolución percibida se describen 43 casos donde los alumnos se describen predominantemente como colaboradores, de los cuales, solo 27 se mantienen en su estilo, sin verse forzados a cambiarlo durante el semestre. El resto, dada su experiencia grupal se vio forzado a desarrollar estrategias de adaptación complementarias: a). 12 casos donde por conflictos en el grupo, los alumnos tuvieron que desarrollar estilos más competitivos, b). 12 casos donde por conflictos en el grupo, los alumnos tuvieron que desarrollar estilos más evitativos, c). 12 casos donde por buen trabajo en equipo, los alumnos asumieron estrategias y estilos más complacientes, d). 7 casos donde por coyunturas, percibieron que tuvieron que desarrollar estilos negociadores.

4.2.1.2. Análisis Cualitativo de Contenido: Una de los sub-grupos que se 
observa, es la evolución de grupos con dinámicas del tipo colaborador, es el paso o tránsito hacia modos complacientes de afrontamiento dentro del grupo. Es el caso del siguiente alumno:

- En mi grupo me sentí cómodo en un principio, aportaba lo necesario y asistía a todas las reuniones, de vez en cuando ayudaba a algún compañero y una que otra vez le hice alguna corrección que creía pertinente. Más adelante surgieron problemas en el equipo debido a que no todos se presentaban, lo que me hizo desmotivarme un poco del grupo y en consecuencia ya no aportaba tanto y tomé el rol de seguir la mayoría...

Dentro de esta misma evolución complementaria, desde lo colaborativo a lo complaciente, narraciones como las del siguiente alumno:

- Mi adaptación en el grupo fue muy buena, nadie se interponer sobre otro, y eso me permitió aportar de la mejor forma. Además, al compartir los mismos intereses y presentar la voluntad de ayudar, el ambiente del grupo fue el óptimo (...). El rol que asumí en mi grupo fue entre pasivo y cooperador, si bien no me gusta exponer mis ideas, aprendí a expresar todas aquellas que me parecían trascendentales y a cooperar con los trabajos que me pidiesen.

También resulta interesante analizar casos de alumnos, que inicialmente se muestran colaboradores, pero en la medida que tienen una experiencia negativa o se les dificulta integrar sus ideas, deciden retraerse:

- En un principio quise hacer lo que más podía, por lo que a mí respecta, y colaborar con todo, tengo imaginación y no soy tan malo para dibujar, pero al darme cuenta de que las ideas de los demás no eran parecidas a las mías, pretendí seguir la corriente y hacer lo que ellos pensaban que era mejor, ya que sus ideas eran buenas y no encontraba que las mías fueran mejores. Esto me hizo dependiente de hace lo que me pedían y no ser un sujeto que da su opinión al grupo, ni mucho menos corregir ideas que creía erróneas, a lo largo 
de los trabajos me empezó a parecer cada vez más tedioso aportar al grupo, cada vez fui más irresponsable, menos preocupado y aportativo, y negativo para el grupo, tanto como la visión que ellos tienen de mí, como consecuencia conseguí un mal cartel propio y el desagrado hacia mí de parte de mi grupo...

En otro caso, un estilo inicialmente colaborador, se vuelve evitativo e incluso indiferente a la disolución del grupo: Finalmente, el grupo se comenzó a desarmar, sus lazos se fueron desfigurando por diferencias individuales que en un principio no influenciaron el desempeño grupal, pero que a la larga terminaron por destruir y afectar el desempeño colectivo.

En otro grupo, se observó una evolución desde rasgos colaborativos a estilos competitivos, como los de la siguiente alumna:

- En mi grupo me sentí al principio muy apoyada con ellos, nos llevábamos todos bien, se veía que a futuro íbamos a tener buenos trabajos, no como los otros grupos que nadie ni sabía con quién estaba, éramos unidos. Pero en la medida que se iban dando más trabajos grupales, nadie tomaba la iniciativa de poder hacer los trabajos, siempre había excusas para empezar algún trabajo, era muy complicado juntarnos. Igual me sentía súper molesta en el sentido que parecía mamá detrás de todos junto con otra compañera, quería poder desarrollar bien los trabajos, pero sentía que no tenían ganas de estar.

Finalmente, también destaca la experiencia de alumnos que, asumiendo actitudes colaboradoras de modo inicial como estrategia principal de inserción dentro del grupo, fueron validados como líderes negociadores:

- Al principio como todo grupo siempre hay personas que no van acorde con nuestro ritmo y que no desean trabajar, aun así, algunas trataron de ayudar, y las que no, solas fueron dejando el grupo. Me sentí cómoda al saber que entre todos hay un gran respeto en cuanto a mis opiniones, tiempos de 
trabajo y organización (...). Siento que al ver cuando se produce un momento de caos es momento de actuar y respetar cada una de las opiniones llevando a una que nos deje a todos satisfechos. Siento que discutiendo sin razón no se llega a ningún lado y solo nos retrasa.

\subsection{Estilo Evitativo:}

4.3.1. Análisis Descriptivo: 17 alumnos se perciben con un estilo predominantemente evitativo, de los cuales, dada su experiencia grupal, desarrollaron estrategias de adaptación complementarias: a). 9 alumnos, dada una experiencia de grupo, logra integrarse de manera colaborativa, b). 4 alumnos ante coyunturas y dificultades asumen estilo negociador, c). 4 alumnos, dadas dificultades en el grupo, se sienten compelidos a desarrollar estilos competitivos más individualistas.

4.3.2. Análisis Cualitativo de Contenido: En la práctica, pareciera que este grupo presenta mayor grado de flexibilidad para asumir diversos roles complementarios, destacando el estilo colaborador dentro del grupo, lo que se muestra en la siguiente descripción:

- Durante los trabajos en grupo de este semestre aprendí a trabajar de distintas maneras. Al comienzo no me agradaba la idea de trabajar en conjunto con otras personas, pero aprendí a hacerlo, ya que tenía otras situaciones y experiencias pasadas en las que unos trabajaban más que otros y bueno, así se dio...

Similar es el caso de un alumno que describe similar situación de base, pero con mayores grados de y evolución hacia formas más complacientes de afrontamiento y adaptación:

- En mi grupo durante el semestre, me sentí "sin pena ni gloria", siempre quise destacar un poco más, mostrar más mis ideas y mi forma de trabajar, que hasta el año pasado lo encontraba bastante eficiente, pero el estar 
trabajando con mi grupo este semestre me ha llenado un tanto de inseguridades, siempre dentro del grupo nunca me involucraba mayormente, en discusiones lo mismo, sólo me hacía a un lado.

También destacan algunos casos, como el siguiente, donde a partir de una actitud más bien evitativa inicial, la alumna logra transitar hacia roles más negociadores, asumiendo un rol más negociador dentro del grupo, a fin de encausar las diferencias que surgen dentro del grupo:

- En un principio, mi rol dentro del grupo de trabajo fue pasiva. Creo que esto sucedió porque el grupo se formó cuando estábamos entrando a la carrera, sin mucha experiencia ni confianza con el resto de los compañeros que componía el grupo. Yo aportaba con ideas, pero quizás sin ser lo suficientemente convincente (...). En ese momento, pienso que mi rol pasó a ser conciliador, buscando terminar con los conflictos armados a partir de las diferencias de opinión y sacando lo mejor de ambas posturas, para así aspirar a un resultado satisfactorio.

También destacan estilos que de ser descritos como evitativos, asociados a rasgos de timidez personal, desarrollan estilos colaboradores, estilos y modos negociadores como variantes complementarias:

- En el grupo en el cual me toco trabajar me sentí súper cómoda y en cierta parte con un rol de conciliadora, ya que siempre tendía a que nos pusiéramos de acuerdo y que a todos nos favoreciera lo que estábamos realizando. Aunque al comienzo, sólo escuchaba lo que los demás decían y acataba las decisiones tomadas por los otros integrantes del grupo, ya que mi personalidad, al verse enfrentada a gente desconocida, se oculta un poco y me hace ser muy tímida. 


\subsection{Estilos Competitivos:}

4.4.1. Análisis Descriptivo: En tercer término, 6 casos de alumnos que se perciben predominantemente como competitivos, de los cuales, dada su experiencia grupal, desarrollaron estrategias de adaptación complementarias: a). 4 alumnos que describiéndose como competitivos, y dada buena experiencia de trabajo grupal, desarrollaron estilos colaboradores, b). 1 alumno que describiéndose como competitivo, dado la mala experiencia de grupo, se vuelve evitativo en su contacto con el grupo, y c). 1 alumno competitivo, que luego asume estilo negociador en el grupo.

4.4.2. Análisis Cualitativo de Contenido: El estilo que aparece como más descrito, y que aparece como forma complementaria en su evolución, durante el semestre, es el estilo colaborativo, el cual, no dejo de ser exento de complejidades y realidad grupal, como a los costos emocionales y personales de asumir. Es el argumento del siguiente caso:

- Trate en la medida de lo posible de aportar y colaborar para que funcionáramos, y aunque en ocasiones prefiero dar mi brazo a torcer para evitar problemas, creo que no fue lo más adecuado ya que generalmente trato de que se llegue a un consenso con las ideas de los otros integrantes, esto es lo que ha ocurrido últimamente...

Similar situación escribe otro alumno, de modo más desagradable, perteneciente a distinto grupo:

- Yo tiendo a trabajar mejor sola, y mi experiencia grupal al principio fue incomoda, difícil, des-motivante, estresante, angustiante, pero al pasar el tiempo, la relación con algunos de mis compañeros mejoró, lo cual permitió más comunicación y cercanía lo que ayudo en parte a mejorar el trabajo en equipo, pero aun así no hemos logrado compenetrar en un 100\%.

También hemos de destacar el estilo evitativo como modo 
complementario al estilo competitivo. En este caso, el alumno está consciente de la dificultad de trabajar en equipo, lo que supone formas más colaborativas, por lo que el trabajo se orienta hacia una forma más eficiente de desempeño individual:

- Para mí el trabajo en equipo es algo difícil ya que estoy acostumbrado a trabajar solo porque me resulta mucho más fácil que tener que estar poniéndome de acuerdo en quién hace en grupo algo o que no siempre trabaja, por eso cuando trabajo en grupo intento hacerlo con gente que tiene el mismo enfoque en lo que se refiere al trabajo. Por ejemplo, el hacer cosas para mí y a veces no logro entender a algunos compañeros que hacen las cosas a último minuto o que ponen problemas para trabajar.

\subsection{Estilo Negociador.}

4.5.1. Análisis Descriptivo: En cuarto término, 10 alumnos se perciben con estilos negociadores, de los cuales, dada su experiencia grupal, desarrollaron estrategias de adaptación complementarias, tales como: a). 8 de ellos, ante buena experiencia de grupo, se complementa con estilos competidores, b). 1 de ellos, dada mala experiencia grupal, se retrae y vuelve evitativo con el grupo, y c). 1 de ellos asume rol negociador ante conflictos.

4.5.2. Análisis de Contenido: Del análisis de gráfico y tabla, se infiere una cierta naturalidad en la evolución y complementariedad de este tipo de estilo de afrontamiento grupal, con formas colaborativas, incluso, en situaciones y coyunturas conflictivas, que intentan resolverse por recuperar la confianza grupal-colaborativa, antes que la imposición. Tómese el siguiente relato:

- Al principio de mi trabajo con mi grupo, todos fueron bastante tímidos y nadie intentaba dar a conocer su opinión, era difícil empezar sin que alguien por iniciativa propia fuera capaz de decirnos como quería que hiciéramos el trabajo. Como yo fui quien intentó hacer fluir los comentarios de los demás e intentaba conciliar las opiniones, me tomaron de referencia para decidir por el 
resto en cosas fundamentales para nuestro trabajo. No diría que fue autoritaria mi posición porque mis compañeros me la otorgaron al sentir que dentro del grupo yo era responsable.

En otro caso asociado a un grupo distinto, un alumno refiere una dinámica que complementa el estilo negociador predominante, con un estilo evitativo: mi desempeño, más bien mi rol dentro del grupo es escuchar a cada uno, y sobre todo sus ideas e intentar conciliar en común acuerdo...

\subsection{Estilo Complaciente.}

4.6.1. Análisis Descriptivo: En quinto término, 10 alumnos se perciben con estilos complacientes, de los cuales, dada su experiencia grupal, desarrollaron estrategias de adaptación complementarias: a). 5 desarrollaron estilos competitivos, ante conflictos grupales, b). 3 desarrollaron estilos colaboradores, c). 1 desarrollo un estilo negociador complementario ante conflictos grupales, d). 1 desarrollo un estilo evitativo complementario ante conflictos grupales.

4.6.2. Análisis de Contenido: Como se observa, una situación tipo dentro de este tipo de estilo, fue el desarrollo de estrategias competitivas e individualistas de adaptación, ante la dificultad de integrarse o no verse identificados con las propuestas dominantes, como el siguiente caso:

- En los trabajos en grupos siempre he sido como obrera, no me importa mucho ser líder ya que me gusta la igualdad (...). pero en nuestro grupo hubo un líder supremo con voz para todo, pero yo y un compañero igual terminamos haciendo el trabajo solos porque ellos siguiendo al líder no lo hacían bien, (Por esto) mi papel dentro del grupo fue de oposición porque como solo el líder tenía opinión y decisión dentro, yo me oponía dando mi parecer.

Otro alumno, de otro grupo de trabajo, describe un patrón general 
similar de adaptación inicial que evoluciona hacia formas más individualistas con rasgos competitivos. Al respecto, el alumno indica que:

- Cuando XXXX asumió el rol de líder, me temo que fue más allá que ser el portavoz ya que comenzó a dar órdenes y además, bueno, hicimos el trabajo y por lo visto la formula funcionó, sacamos buena nota, por lo tanto seguimos esa orgánica de trabajo (en mi opinión errónea) ya que nuestros compañeros sólo acataban órdenes y no desarrollaban un emprendimiento y una creatividad que nuestra carrera nos exige.

También destaca la descripción de una evolución que complementa un estilo complaciente con estrategias colaborativas: Durante el semestre mi posición en el grupo fue complaciente, preferí en la gran mayoría de los casos ceder a las ideas de otros para evitar discusiones y ocupar ese tiempo en desarrollar el trabajo de manera más rápida.

\section{Conclusiones}

Se pueden exponer en los siguientes puntos:

5.1. El principal grupo de base, con el cual un porcentaje significativo se identificó como estilo de afrontamiento primario, fue el estilo colaborativo (52\%), siguiéndoles el estilo evitativo (24\%), y complaciente (12\%), negociador (8\%) y competitivo (7\%).

5.2. El estilo complementario secundario en cambio sufre variaciones significativas, donde destaca que: el estilo colaborador presenta un $31 \%$, seguido del estilo competitivo (24\%) y evitativo (18\%), negociador (16\%) y complaciente $(11 \%)$.

5.3. Una parte importante de estos estilos secundarios tiende a asumir estrategias competitivas (24\%), en la medida que se acentúa un rasgo individual, o bien, perseverando en estrategias más grupales de colaboración (32\%), pese a las dificultades grupales descritas- y en menor medida de 
incluso mediación o negociación (11\%).

5.4. Por otro lado, narrativamente, destaca un grupo amplio, que se autodefine en general, como pasivo, y que en la descripción narrativa oscila entre rasgos evitativos (16\%) y complacientes (11\%) que sumados hacen un $26 \%$.

5.5. De lo anterior, destaca en primer término, el desarrollo de estilos secundarios, en el siguiente orden: a). colaborador (32\%), tiende a ser competitivo (24\%), evitativo (18\%) y complaciente (16\%). Se sugiere que, en los estilos competitivos, complacientes y evitativos, esta evolución está influenciada por procesos de deterioro grupal -con el surgimiento de conflictos intragrupales- o una posterior auto-desvinculación en algún grado o medida de parte de ellos, forzándolos a trabajar por sí mismo, a fin de compensar dicha brecha, y asumir una actitud evitativa.

\section{Reflexiones y Síntesis Final. \\ Destacan los siguientes puntos:}

6.1. Se desprende de los análisis de contenidos, que para muchos alumnos, resulta un trabajo complejo y de alto costo emocional, el adaptarse a las dinámicas grupales, en cualquiera de los estilos de afrontamiento que desarrollen, como complementarios a los personales primarios y dominantes. Destacan así, como positivo, este tipo de instancias de reflexión, donde los alumnos puedan incorporar niveles de reflexión y auto observación, evitando que se generalice meramente a partir de una buena o mala experiencia individual en un grupo, rigidizando sus posturas y perdiendo flexibilidad ante futuras experiencias individuales y grupales.

6.2. En lo particular, destaca la escasa forma espontánea de estilos negociadores que puedan canalizar dentro del propio grupo, las diferencias. Esto sugiere, que de mantenerse esta tendencia en próximos años, pudiera requerirse de la incorporación de estrategias guiadas que potencian roles mediadores o negociadores en este sentido. 
6.3. También se percibe una disminución espontanea respecto del estilo competitivo como modo primario, como modo de compensar las falencias integrativas y crecientes grados de eficacia del grupo.

6.4. Respecto a los estilos individualistas, no solo destacan los modos competitivos, sino también los evitadores y complacientes. Esto sugiere el potencial aumento de reacciones estresantes como una cierta dificultad de negociar o consensuar ideas, que se compensa con la sensación pragmatista orientada al fin. Esta dinámica donde el trabajo grupal potencia estilos competitivos, evitativos o complacientes sin duda conlleva una suerte de paradoja, pues las ideas no se resuelven ni desarrollan grupalmente, sino por la exigencia de algunos sobre otros, lo cual supone grados de estrés personal significativos, tanto para quienes imponen unas ideas, como para quienes las aceptan evitativa o complacientemente por razones pragmáticas vinculado a la necesidad de eficiencia y toma de decisiones.

\section{Referencias}

Kilmann, T. (1974). Conflict and conflict management. Ed. Palo Alto. Kilmann, R.H. \& Thomas, K.W. (1977). Developing a forced choice measure of conflict handling behavior: The "MODE" instrument. Educational and Psychological Measurement, In: Journal of Management 37: págs. 309-325.

Piorno, M. (2012). Estilos de afrontamiento al conflicto para la eficiencia organizativa en equipos de Enfermería de Atención Primaria. Tesis Magister en enfermería, España: Universidad de Barcelona. Recuperado de:

http://diposit.ub.edu/dspace/bitstream/2445/32202/1/estilos.pdf

Shakespeare, William (1969). Sueño de Una Noche de Verano. En: Obras Selectas. Ed. BAC. 


\section{Omar Eduardo Cañete Islas \\ e-mail: ocanetei00@yahoo.es}

Nacido en Chile. Docente Escuela de Arquitectura.

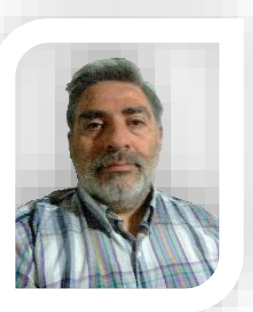

Universidad de Valparaíso, Chile. Magister en

Psicología Social. Docente carrera de Arquitectura desde 2000 a la fecha. Trabajos en temas de forma, territorio y ciudad, además de procesos creativos.

Diversos artículos en revistas indexadas de

especialidad.

El contenido de este manuscrito se difunde bajo una Licencia de Creative Commons Reconocimiento- 